\title{
Functional Evaluation of Anterior Cruciate Ligament Reconstruction - Comparison of Two Graft Fixation Techniques in the Femoral Tunnel
}

\author{
S. Vijayan, S. M. Cherukuri, M. S. Kulkarni, M. A. Naik, S. Shetty, V. Bhat, S. D. Murali, \\ S. K. Rao
}

Department of Orthopedics, Kasturba Medical College, Manipal Academy of Higher Education, Manipal, India

\author{
CORRESPONDING AUTHOR: \\ Mahesh Suresh Kulkarni \\ Department of Orthopedics \\ Kasturba Medical College - Manipal \\ Manipal Academy of Higher Education \\ Udupi, Karnataka, India 576104 \\ E-mail: maheshskulkarnibmc@gmail.com
}

DOI:

10.32098/mltj.01.2021.07

LEVEL OF EVIDENCE: 3B

\begin{abstract}
SUMMARY
Background. There are several fixation techniques and devices available for ACL reconstruction, which differ significantly in their efficacy. There are no studies in the literature comparing femoral transfixation pins (Rigidfix) and adjustable loop (Rigidloop) devices. We compared the outcomes of these devices in our study.

Methods. A retrospective observational cohort study was done between November 2017 and June 2019. Of the total 95 cases, initial 47 patients had undergone graft fixation with Rigidfix and the subsequent 48 underwent fixation with Rigidloop. Clinical and functional evaluation of these patients was done at one-year follow-up and the results were compared.

Results. The two groups were homogenous demographically. Though better outcome and functional scores were noted in the Rigidfix group, these observations were not statistically significant. Two Rigidloop cases had side to side difference in KT-1000 reading of $>5 \mathrm{~mm}$ with a fair outcome.

Conclusions. Fixation of the hamstring autograft on the femoral side using Rigidfix cross pin and Rigidloop adjustable suspensory devices provide statistically comparable stability. The Lysholm grading and IKDC-2000 functional scores between both the groups were similar at one-year follow-up and hence can be used interchangeably intraoperatively if a need arises without any consequences.
\end{abstract}

KEY WORDS

ACL reconstruction; adjustable loops; cross pins; KT-1000 test; Rigidfix.

\section{BACKGROUND}

The outcome of ACL reconstruction can be influenced by many factors like the experience of the surgeon, graft characteristics, orientation of the bony tunnels, technique of drilling the femoral tunnel (transtibial or transportal), graft fixation technique, postoperative rehabilitation protocol and period of assessment (confounding variables) (1). Over the past few decades, as the understanding of the anterior cruciate ligament (ACL) reconstruction techniques changed, so did the choice of graft and fixation devices (2-5). Hamstring autograft has emerged as the preference of most surgeons over the years (2). An ideal graft fixation device should be strong enough to avoid failure, stiff enough to restore knee stability and secure enough to avoid slippage $(1,6-8)$.
It's preferable that it is magnetic resonance imaging (MRI) compatible and if it's bioabsorbable then it will allow easy revision $(8,9)$.

The search for this ideal graft fixation device has led to the discovery of several newer implants and techniques over the years, but still, there is no accepted gold standard (2, 8, 10). Again, there are several implants available in the market acting by the same fixation principle which differs significantly in their efficacy as proven by various studies $(2,4,6,11)$.

\section{PURPOSE OF THE STUDY}

Literature comparing the outcome of fixation techniques using femoral transfixation pins and adjustable suspenso- 
ry loops is sparse. Moreover, as per our knowledge, there are no studies in the literature comparing Rigidfix (femoral transfixation pins by Depuy Mitek, USA) and Rigidloop (adjustable loop by Depuy Mitek, USA) devices. Only one similar study was found, done by Zehir et al., which compared the outcome between Translig (transfixation pins) and ToggleLoc (adjustable loop) devices (12). This study had some stark limitations like variable femoral tunnels and inconsistency in graft fixation devices used at the tibial end. In our study, we compared the outcomes of Rigidfix and Rigidloop fixation devices by addressing these limitations. We hypothesized that both Rigidfix and Rigidloop will provide similar clinical and functional outcomes. Our primary objective was to compare anteroposterior and rotational instability. We used Lachman's test and KT-1000 for measuring AP instability. The pivot shift test and hop limb symmetry index assessed the rotational instability. Lysholm score and IKDC-2000 scoring were used to evaluate the functional outcome which was our secondary outcome measure.

\section{MATERIALS AND METHODS}

A retrospective observational cohort study was done between November 2017 and June 2019. Institutional Ethical Committee (IEC NO: 19/2018) clearance was obtained and study protocol was as per STROBE guidelines. Data of skeletally mature patients who underwent ACL reconstruction from November 2017 to June 2018 were collected and patients were divided into two groups based on the femoral fixation technique. A total of ninety-five cases were identified after applying the inclusion-exclusion criteria. There were 47 patients who underwent graft fixation at the femoral side with Rigidfix and 48 patients who underwent fixation with Rigidloop. To minimize the effect of the multiple confounding variables, all cases in our study were operated by a single surgeon who was equally adept in both the fixation techniques. The graft characteristics and tunnel creation were also standardized by using single bundle quadrupled hamstring autologous graft and tunnels were created using the transportal technique in both groups. In all patients, the tibial fixation was achieved using an Intrafix (Depuy Mitek) screw with sheath. All patients underwent the same rehabilitation protocol and were clinically and functionally evaluated at one year after the index procedure. Patients who had concomitant collateral or posterior cruciate ligament injury, tibial plateau avulsion fractures, bilateral ACL injuries and those undergoing revision ACL procedures were excluded from this study. Clinical outcome was evaluated by Lachman, pivot shift and single-leg hop test. For the single- legged hop test, the subjects stood on the leg to be tested, hopped and landed on the same limb. The distance hopped was measured from the tip of the great toe of one step to the other step of the same foot and recorded in centimetres. Single hop for distance was quantified as hop limb symmetry index (LSI) which was expressed as the percentage of the longest 'involved' limb hop distance divided by the longest 'uninvolved' limb hop distance (13). KT-1000 arthrometer readings were also taken for an objective measure of anteroposterior translation of tibia. KT-1000 arthrometer values, were recorded at $20 \mathrm{lbs}$ and side to side difference was calculated (values expressed as operated - healthy knee difference). The side to side difference was calculated to neutralize the laxity, if at all, in the uninvolved knee. These values were categorized into three groups: $<3 \mathrm{~mm}, 3-5 \mathrm{~mm}$, and $>5 \mathrm{~mm}$ side to side difference $(4,14)$. Functional performance was assessed by Lysholm knee scoring scale and IKDC-2000 subjective knee evaluation score. Lysholm knee scoring scale comprises of eight parameters for evaluation (15). The maximum possible knee function score is 100 . The outcome scores were graded as excellent (>90), good (84-90), fair (65-83) and poor $(<65)$. IKDC-2000 subjective evaluation has 10 questions and 18 responses (16). The total score which ranges from 0 to100 was calculated as (sum of individual item score/87) x 100 .

\section{STATISTICAL METHODS}

Statistical analysis was done in IBM ${ }^{\circledR}$ SPSS version 25 software. Demographic data were presented using descriptive statistics. Categorical variables such as clinical test grades (Lachman and Pivot shift tests), Lysholm knee score and KT-1000 arthrometer measurements were analyzed using Chi-square or Fisher's exact test where appropriate. Continuous variables of hopped limb symmetry index (LSI) and IKDC-2000 scores were analyzed by independent sample $t$ test. The difference in mean IKDC-2000 scores between the groups was calculated using the one-way ANOVA test. In all these analytics, the P-value of $<0.05$ was considered statistically significant. P-value was calculated with a $95 \%$ confidence interval and an alpha error of $5 \%$. Power analysis was done from Open Epi, Version 3, open-source calculator.

\section{RESULTS}

All patients were analyzed at 1-year from index procedure. Our study groups were comparable with respect to key demographic and constitutional variables (table I).

Clinically, patients in Rigidfix group showed greater anteroposterior stability in comparison to those in Rigidloop group, as noted by the Lachman grading and KT-1000 readings (table II). However, this difference was not statistical- 
ly significant. $(\mathrm{P}=0.22$ and $\mathrm{P}=0.56$ respectively). In our study, we had 2 patients in Rigid loop group with KT-1000 differential (side to side) displacement of $>5 \mathrm{~mm}$ which was considered as the failure of graft fixation.

On a similar line, grade 1 positive pivot shift was observed in 3 patients of Rigidfix group and 10 patients of Rigidloop group. However, this difference was statistically insignificant $(P$-value $=0.07)$. None of the patients from either group had grade 2 or 3 Pivot shift (table II). Also, the average hop limb symmetry index (LSI) scores for both groups were similar (table II) and not significant statistically $(\mathrm{P}=0.72)$. The differences in the Lysholm knee scoring scale between the two groups were insignificant $(\mathrm{P}=0.18)$ (table II). We had 4 poor outcomes as per the Lysholm grading in the Rigid loop group. Mean IKDC-2000 scores between both the groups were similar (table II).

Patients who had meniscal injuries, irrespective of the fixation technique had an overall significantly worse IKDC2000 score. Patients in Rigidloop group had significantly worse IKDC-2000 scores when associated with meniscal injuries (table II). We had 2 failures as per the KT-1000 measurements which were $>5 \mathrm{~mm}$ side to side difference. Both the patients had fair outcome in Lysholm grading. One of them demonstrated grade 1 laxity on Lachmann's and pivot shift test while the other showed grade 1 laxity on Lachmann's and grade 0 on Pivot shift test.

Our study had a sample size of 47 and 48 in each group. The upper limit of reference value for excellent and good outcome in Tegner Lysholm Knee scoring scale is 100 and 90 respectively. Presuming that two groups would show a difference of 10 points and with the standard deviation of 16 (difference of upper and lower end of the range), our study had the power of $86.12 \%$ with an alpha error of $5 \%$.

\section{DISCUSSION}

We noted that the static and dynamic stability tests and functional outcome scores did not show statistically significant difference on using Rigidfix or Rigidloop for anchoring the hamstring graft in the femoral tunnel. It takes around 3-6 months for complete osteointegration of the hamstring graft to the host bone. This 'weak link period' is the time during which we allow unrestricted weight-bearing, full range of motion and early return to strenuous day-to-day activities $(2,17)$. It is at this critical phase that the graft fixation device is required to provide secure anchorage until biological fixation (graft integration into the host) takes place (14). Therefore, it is extremely important that the fixation device being used should provide adequate stability to the graft to withstand the forces which are being generated during the accelerated postoperative rehabilitation programs employed these days $(1,4,10)$.

Three types of fixation techniques are practised worldwide, namely aperture fixation (direct fixation - e.g., interference screw), cortico-cancellous transfixation (e.g., cross pins) and cortical suspensory fixation (indirect fixation - e.g., fixed and adjustable loops with button) $(3,8,18)$. Each of these techniques have their own advantages and disadvantages $(1,19)$. Many studies are available in literature which has compared these different fixation techniques and devices (2, 10, 20, 21). However, there is still no consensus about the best fixation technique and the market is flooded with several types of fixation devices $(4,10)$.

The working length of the graft is between the two points of its fixation in the tibial and femoral tunnels. A shorter working length by means of an intra-tunnel fixation will be more stiff and provide better stability than when the graft is fixed outside the tunnel which increases the working length $(3,8)$. Interference screw which gives adequate initial stability can become loose, damage the hamstring graft and

Table I. Demographic distribution and comparison of both the groups.

\begin{tabular}{|c|c|c|c|c|}
\hline \multirow{2}{*}{ Parameter } & \multicolumn{2}{|c|}{ Fixation technique } & \multirow{2}{*}{ Total } & \multirow{2}{*}{ P-value } \\
\hline & Rigidfix & Rigidloop & & \\
\hline Number & 47 & 48 & 95 & - \\
\hline Mean age (years) & $33.02 \pm 8.8$ & $32.9 \pm 9.5$ & $33 \pm 9.1$ & 0.98 \\
\hline Male:Female & $37: 10$ & $40: 8$ & $77: 18$ & 0.60 \\
\hline Right:Left & $21: 26$ & $24: 24$ & $45: 50$ & 0.68 \\
\hline $\begin{array}{l}\text { Common mode of injury } \\
\text { Fall:RTA:Sports Injury }\end{array}$ & $13: 19: 15$ & $15: 21: 12$ & $28: 40: 27$ & 0.78 \\
\hline $\begin{array}{l}\text { Meniscal injuries } \\
\text { (number of cases) }\end{array}$ & 8 & 15 & 23 & 0.08 \\
\hline
\end{tabular}

RTA: Road Traffic Accident 
Table II. Comparison of clinical and functional outcome of both the groups.

\begin{tabular}{|c|c|c|c|c|c|}
\hline \multirow[b]{2}{*}{ Clinical test } & \multirow[b]{2}{*}{ Grading } & \multicolumn{2}{|c|}{ Fixation technique } & \multirow[b]{2}{*}{ Total } & \multirow[b]{2}{*}{ P-value } \\
\hline & & $\begin{array}{l}\text { Rigid fix } \\
(n=47)\end{array}$ & $\begin{array}{c}\text { Rigid loop } \\
(n=48)\end{array}$ & & \\
\hline \multirow{3}{*}{ Lachmann's test } & Grade 0 & 38 & 35 & 73 & \multirow{3}{*}{0.22} \\
\hline & Grade 1 & 9 & 11 & 40 & \\
\hline & Grade 2 & 0 & 2 & 2 & \\
\hline \multirow{3}{*}{$\begin{array}{l}\text { KT-1000 values at } 20 \mathrm{lbs} \text { side to side } \\
\text { difference }\end{array}$} & $<3 \mathrm{~mm}$ & 36 & 34 & 70 & \multirow{3}{*}{0.56} \\
\hline & $3-5 \mathrm{~mm}$ & 11 & 12 & 23 & \\
\hline & $>5 \mathrm{~mm}$ & 0 & 2 & 2 & \\
\hline \multirow{2}{*}{ Pivot shift test } & Grade 0 & 44 & 38 & 82 & \multirow{2}{*}{0.07} \\
\hline & Grade 1 & 3 & 10 & 13 & \\
\hline Hop limb symmetry index & & $84.26 \pm 11.85$ & $85.19 \pm 13.65$ & & 0.72 \\
\hline \multirow{4}{*}{ Lysholm Score } & $\begin{array}{l}\text { Excellent } \\
(>90)\end{array}$ & 38 & 37 & 75 & \multirow{4}{*}{0.18} \\
\hline & $\begin{array}{c}\text { Good } \\
(84-90) \\
\end{array}$ & 1 & 0 & 1 & \\
\hline & $\begin{array}{c}\text { Fair } \\
(65-83)\end{array}$ & 8 & 7 & 15 & \\
\hline & $\begin{array}{l}\text { Poor } \\
(<65)\end{array}$ & 0 & 4 & 4 & \\
\hline \multicolumn{2}{|l|}{ IKDC-2000 scores } & $89.52 \pm 11.82$ & $84.30 \pm 15.45$ & & 0.068 \\
\hline \multicolumn{2}{|l|}{$\begin{array}{l}\text { IKDC-2000 scores with } \\
\text { Meniscal injury }\end{array}$} & $85.09 \pm 13.22$ & $69.33 \pm 16.34$ & $74.81 \pm 16.86$ & 0.023 \\
\hline \multicolumn{2}{|l|}{$\begin{array}{l}\text { IKDC-2000 scores without } \\
\text { Meniscal injury }\end{array}$} & $90.43 \pm 11.48$ & $91.10 \pm 9.00$ & $90.74 \pm 10.35$ & 0.78 \\
\hline \multicolumn{2}{|l|}{$\begin{array}{l}\text { P-value (comparison of IKDC- } \\
2000 \text { score with or without } \\
\text { Meniscal injury) }\end{array}$} & 0.315 & 0.000 & 0.000 & \\
\hline
\end{tabular}

increase the risk of posterior blowout with resultant failure of fixation (12). Cortico-cancellous pins give adequate graft fixation but have issues related to breakage, migration and prominence and minimal tunnel widening $(2,3$, $8,19,22)$. Suspensory devices (fixed and variable loops) have issues related to longitudinal and sagittal graft motion (bungee and windshield wiper effect respectively) within the tunnel leading to widening of the tunnel and loosening of the fixation $(7,19,23)$. Tunnel widening is reported to be greater on the femoral side than on the tibial side (21). Yet another contention for the adjustable loop design is loop lengthening occurring due to loosening of the knot $(1,6,7,10,12,14)$. Graft motion can lead to extravasation of synovial fluid between graft and bone tunnel and increase the cytokine levels within the knee which could further delay the tendon-bone healing $(2,19,23)$. Volume rendered 3D imaging modality is considered superior than plain radiographs, MRI and SPECT (Single Positron Emis- sion Tomography) scan in evaluating bone tunnel widening after ACL reconstruction $(24,25)$.

Eajazi et al. (17) compared the three basic techniques of graft fixation and found that cortico-cancellous method provided the optimal fixation and stiffness. The weakest fixation was with the cancellous device and cortical suspension device demonstrated variable mechanical behaviour.

Rigidfix (Depuy Mitek, Raynham, MA) uses two pins across the graft and the femoral tunnel and creates a bulge in the graft. It reduces the risk of posterior tunnel blow out and decreases the windshield wiper effect as it fixes the graft horizontally preventing mobility between graft pins ( 8 , 17). The resistance which is distributed along the surface between the device and the bone depends on the osseous density and length of the lever arm (17). Angel et al. and Basad et al. demonstrated that cross pin fixation when compared to Endobutton provided superior anteroposterior stability as measured with KT-1000 (18). However, Price 
et al. and Basad et al. found no significant difference in the clinical and functional outcome between the two groups $(18,26)$. In our study, the majority of patients in the Rigidfix group had excellent anteroposterior stability as evidenced by the negative Lachman test $(38 / 47$ or $80 \%)$ and objective KT-1000 arthrometer readings (36/47 or $77 \%$ ). Ninety-four percent (44/47) of the patients had restored rotational stability as seen by a negative pivot shift test and there were no patients with grade II or III pivot shift in the Rigidfix group. The dynamic stability of the affected limb was restored to nearly $85 \%$ of the normal limb as indicated by the hop limb symmetry index. At the end of one-year postop, more than 90 percent $(38 / 47)$ of patients were noted to have an excellent functional outcome. Asik et al., Nebelung et al. and Choi et al. reported femoral tunnel widening in patients with broken or dislocated cross pins $(9,22$, 27). However, it did not affect the functional outcome. They were not able to give a valid reason for the occurrence of the tunnel widening though they felt it was due to improper graft-bone healing (22).

Many authors had compared fixed and adjustable loop suspension devices $(6,7,14)$. Boyle et al. found no significant difference in the postoperative knee stability between the groups and commented that adjustable loop devices do not clinically loosen during rehabilitation (14). Transportal technique creates more anatomical tunnel in the femur, but has the risk of producing shorter tunnel. The fixed loop devices leave a dead space in the tunnel which is occupied only by the loop. In contrast, the adjustable loop devices allow to pull greater length of the graft into the tunnel and thereby negates the dead space. This achieves more grafthost bone contact and better osteointegration, especially in the shorter tunnels (14). In vitro studies comparing the biomechanical properties between the two cortical suspension devices showed that both devices can withstand the forces developed during rehabilitation, but the adjustable loop shows mild elongation without clinical significance (6, $28)$. In our study, we saw that $23 \%$ of the patients (11/48) in the Rigidloop group had grade I Lachmann and 4\% (2/48) had grade II Lachmann laxity. We presume that the recurrence of laxity might have occurred due to the elastic elongation of the graft or the variable loop device as mentioned in the in vitro studies. Similar results were seen in objective measurements using KT-1000 arthrometer also. We had 2 patients in Rigid loop group who had side to side difference in KT-1000 values $>5 \mathrm{~mm}$ suggestive of failure due to graft elongation/slippage. They both had grade 1 Lachmann's, grade 1 and grade 0 pivot shift, and Lysholm grading of fair and IKDC-2000 scoring of 56. However, despite clinical evidence of an increase in laxity, the functional outcomes were excellent at one-year follow-up which was observed by other authors as well (14). Eighty percent of the patients (38/48) had restored rotational stability as seen by a negative Pivot shift test. There were no patients with grade II or III pivot shift. Hop limb symmetry index showed a mean of $85 \%$ in this group.

Some authors have also compared the variable loop devices manufactured by different companies as these devices use different loop locking mechanisms (11). The Zip loop (Biomet) and Tightrope (Arthrex) rely on a finger trap locking mechanism where one end of the adjusting suture is spliced through the centre of the other suture end. When a load is applied in the opposing direction, the finger trap collapses around the inner suture, trapping it in place. Small micromotions can gradually disengage the collapsed finger trap and loosen the locking mechanism. In contrast, the Rigidloop device has a one-way sliding lock that wedges into the metal implant and resists disengagement. The tighter the knot is cinched, the better is the performance. Failure of this mechanism will occur if the knot is left loose (11). The knot-based design outperformed the finger trap designs in both cyclic displacement and strength (11).

After a thorough literature search, we found that there were no studies which had compared Rigidfix (a cortico-cancellous transfixation device) with Rigidloop (an adjustable suspensory loop with button). A similar study was done by Zehir et al., where they compared ToggleLoc with ZipLoop and Translig transverse cross pin devices (12). Although insignificant, the KT-1000 arthrometer revealed slightly less anterior sliding in Translig group than that in the ToggleLoc group $(\mathrm{P}>0.05)$. On the contrary, in our study although statistically insignificant, Rigidfix seemed to provide better anteroposterior stability as evidenced by the results of the Lachman and KT-1000 arthrometer readings. Additionally, we noted that the rotational and dynamic stability as measured by the pivot shift test and hop limb symmetry index were comparable among the groups. As reported in their study, we also observed that the Lysholm and IKDC functional scores between our groups (Rigidfix and Rigidloop) were comparable. Our study confirmed the hypothesis that both Rigidfix and Rigidloop devices provide equally good clinical and functional outcomes at 1 -year follow-up period.

During the analysis, we observed that irrespective of the technique (Rigidfix or Rigidloop), the patients with isolated ACL injuries had significantly better IKDC scores compared to those with concurrent meniscal injuries (table II). It was noted that patients in Rigidloop group had greater worsening of these scores. However, the statistical significance of the poorer IKDC score noted in the patients with meniscal injuries in the Rigidloop group was not assessed due to the small sample size. Similarly Lysholm score differences 
between those with meniscus and those without meniscal injuries were not calculated due to insufficient sample size in each category.

Rigidfix gives cortico-cancellous fixation whereas Rigidloop gives cortical fixation. Though they works by a different mechanism at different locations of the femoral tunnel; the clinical and functional outcomes were comparable with both these devices. This is contrary to the outcome reported in few of the biomechanical and clinical studies $(3,8)$. This can be explained based on the varying supraphysiological loads applied and different biomechanical set up in the in vitro studies at which these devices fail $(4,7,14)$. The physiological load during rehabilitation which will be acting for a limited period, i.e, 3 to 6 month postoperatively during which healing of the graft takes place, will be within the tolerance limit of these devices $(6,8)$.

One of the implications of this study is that Rigidfix and Rigidloop can be used interchangeably without any compromise in the outcome. Various causes for intraoperative on-table failure of cortico-cancellous and cortical devices have been reported in the literature like pin breakage, blowout of posterior and lateral cortex of the femoral tunnel, eccentric placement of crosspins, sinking of button and failure of loop, suture or button $(4,7,8,29)$. With minimal modification in the technique, the surgeon can decide intraoperatively to use either of the methods if the need arises without worrying about the final outcome. This has been our experience in few of the earlier cases which prompted us to conduct this study.

\section{Strength of the study}

There have been no studies in the English literature which have compared the results of graft fixation in the femoral tunnel using the cortico-cancellous transfixation with adjustable loop devices uniformly. In our study, all cases were performed by a single senior surgeon who is well versed in performing both techniques and we have tried to standardize the patient characteristics, tunnel creation method, graft preparation, graft length inside the femoral tunnel, graft fixation on the tibial side and post-operative rehabilitation protocol so that the confounding factors are minimized. Our study was adequately powered to detect a 1-grade difference in the Lysholm scoring system.

\section{Limitations}

Our study had a few limitations. No postoperative radiological analysis of the distal femur was done to look for tunnel characteristics (obliquity, widening) and graft lengthening (23). The latter two are known complications of suspenso- ry fixation devices. Studies have shown no effect of tunnel widening on the outcome $(21,23)$. Hence we did not find it necessary to assess the tunnel characteristics during follow up. Secondly, ours was a short-term study and therefore the long-term effect of ACL reconstruction in preventing further meniscal injuries and cartilage damage and thereby early degenerative arthritis could not be commented. Also, the long-term effect of partial meniscectomy in combination with ACL reconstruction on the function of the knee could not be assessed. Long term follow-up studies are required to assess the effect of these interventions. Thirdly, ours being a retrospective study, preoperative functional scores were not available. Ideally, a randomized controlled study would acquire these baseline scores against which the post-operative functional scores can be compared to analyze the effect of surgical intervention.

In view of significant worse functional outcome seen with patients who had concomitant meniscal injury irrespective of the fixation technique, further studies are required. As we had less number of cases of meniscal injuries and as it was not our objective we refrain from drawing a conclusion on this finding. High-quality randomised controlled studies which a have high number of cases and uniform surgical techniques with long term functional outcome can give verdict regarding the effect of concomitant meniscal injuries on ACL reconstruction. The study was conducted according to the journal's guidelines (30).

\section{CONCLUSIONS}

In view of the similar outcome which we observed in primary and secondary objectives of our study, we accepted the null hypothesis. Hence we conclude that Rigidfix and Rigidloop have a similar clinical and functional outcome at the end of the 1 year and can be used interchangeably if an introperative need arises without any consequences.

\section{CONFLICT OF INTERESTS}

The authors declare that they have no conflict of interests.

\section{REFERENCES}

1. Mayr R, Heinrichs CH, Eichinger M, Coppola C, Schmoelz W, Attal R. Biomechanical Comparison of 2 Anterior Cruciate Ligament Graft Preparation Techniques for Tibial Fixation: Adjustable-Length Loop Cortical Button or Interference Screw. Am J Sports Med 2015;43(6):1380-5.

2. Persson A, Kjellsen AB, Fjeldsgaard K, Engebretsen L, Espehaug B, Fevang JM. Registry Data Highlight Increased Revision Rates for Endobutton/Biosure HA in ACL Reconstruc- 
tion With Hamstring Tendon Autograft: A Nationwide Cohort Study From the Norwegian Knee Ligament Registry, 20042013. Am J Sports Med 2015;43(9):2182-8.

3. Ibrahim SAR, Abdul Ghafar S, Marwan Y, et al. Intratunnel Versus Extratunnel Autologous Hamstring Double-Bundle Graft for Anterior Cruciate Ligament Reconstruction: A Comparison of 2 Femoral Fixation Procedures. Am J Sports Med 2015;43(1):161-8.

4. Houck DA, Kraeutler MJ, McCarty EC, Bravman JT. FixedVersus Adjustable-Loop Femoral Cortical Suspension Devices for Anterior Cruciate Ligament Reconstruction: A Systematic Review and Meta-analysis of Biomechanical Studies. Orthop J Sports Med 2018;6(10):232596711880176.

5. Zhang S, Xu M, Wang X, Wang J. Functional outcome of en bloc excision and custom prosthetic replacement for giant cell tumor of the distal radius. J Orthop Sci 2015;20(6):1090-7.

6. Chang MJ, Bae TS, Moon Y-W, Ahn JH, Wang JH. A Comparative Biomechanical Study of Femoral Cortical Suspension Devices for Soft-Tissue Anterior Cruciate Ligament Reconstruction: Adjustable-Length Loop Versus Fixed-Length Loop. Arthrosc J Arthrosc Relat Surg 2018;34(2):566-72.

7. Cheng J, Paluvadi SV, Lee S, Yoo S, Song E-K, Seon J-K. Biomechanical comparisons of current suspensory fixation devices for anterior cruciate ligament reconstruction. Int Orthop 2018;42(6):1291-6.

8. Rodríguez C, García TE, Montes S, Rodríguez L, Maestro A. In vitro comparison between cortical and cortico-cancellous femoral suspension devices for anterior cruciate ligament reconstruction: implications for mobilization. Knee Surg Sports Traumatol Arthrosc 2015;23(8):2324-9.

9. Nebelung S, Deitmer G, Gebing R, Reichwein F, Nebelung W. Anterior cruciate ligament reconstruction using biodegradable transfemoral fixation at 5-year follow-up: clinical and magnetic resonance imaging evaluation. Knee Surg Sports Traumatol Arthrosc 2012;20(11):2279-86.

10. Monaco E, Fabbri M, Lanzetti RM, Del Duca A, Labianca L, Ferretti A. Biomechanical comparison of four coupled fixation systems for ACL reconstruction with bone socket or full-tunnel on the tibial side. The Knee 2017;24(4):705-10.

11. Pasquali M, Plante MJ, Monchik KO, Spenciner DB. A comparison of three adjustable cortical button ACL fixation devices. Knee Surg Sports Traumatol Arthrosc 2017;25(5):1613-6.

12. Zehir S, Zehir R. Suspensory fixation versus novel transverse crosspin for femoral fixation in anterior cruciate ligament reconstruction. Arch Orthop Trauma Surg 2014;134(11):1579-85.

13. Noyes FR, Barber SD, Mangine RE. Abnormal lower limb symmetry determined by function hop tests after anterior cruciate ligament rupture. Am J Sports Med 1991;19(5):513-8.

14. Boyle MJ, Vovos TJ, Walker CG, Stabile KJ, Roth JM, Garrett WE. Does adjustable-loop femoral cortical suspension loosen after anterior cruciate ligament reconstruction? A retrospective comparative study. The Knee 2015;22(4):304-8.

15. Tegner Y, Lysholm J. Rating systems in the evaluation of knee ligament injuries. Clin Orthop 1985;(198):43-9.

16. Irrgang JJ, Anderson AF, Boland AL, et al. Development and Validation of the International Knee Documentation Committee Subjective Knee Form. Am J Sports Med 2001;29(5):600-13.
17. Eajazi A, Madadi F, Madadi F, Boreiri M. Comparison of Different Methods of Femoral Fixation Anterior Cruciate Ligament Reconstruction. Acta Med Iran 2013;51(7):444-8.

18. Basad E, Kipper A, Wüsten OA, Stürz H, Ishaque BA. Comparative study after hamstring ACL plasty with RigidFix (pin fixation) and EndoButton (anchor fixation). Z Orthopadie Unfallchirurgie 2010;148(3):276-81.

19. Mermerkaya MU, Atay OA, Kaymaz B, Bekmez S, Karaaslan F, Doral MN. Anterior cruciate ligament reconstruction using a hamstring graft: a retrospective comparison of tunnel widening upon use of two different femoral fixation methods. Knee Surg Sports Traumatol Arthrosc 2015;23(8):2283-91.

20. Milano G, Mulas PD, Ziranu F, Piras S, Manunta A, Fabbriciani C. Comparison Between Different Femoral Fixation Devices for ACL Reconstruction With Doubled Hamstring Tendon Graft: A Biomechanical Analysis. Arthrosc J Arthrosc Relat Surg 2006;22(6):660-8.

21. Giorgio N, Moretti L, Pignataro P, Carrozzo M, Vicenti G, Moretti B. Correlation between fixation systems elasticity and bone tunnel widening after ACL reconstruction. MLTJ 2016;6(4):467-72.

22. Choi N-H, Son K-M, Yoo S-Y, Victoroff BN. Femoral Tunnel Widening After Hamstring Anterior Cruciate Ligament Reconstruction With Bioabsorbable Transfix. Am J Sports Med 2012;40(2):383-7.

23. Lanzetti RM, Monaco E, De Carli A, et al. Can an adjustable-loop length suspensory fixation device reduce femoral tunnel enlargement in anterior cruciate ligament reconstruction? A prospective computer tomography study. The Knee 2016;23(5):837-41.

24. de Beus A, Koch JE, Hirschmann A, Hirschmann MT. How to evaluate bone tunnel widening after ACL reconstruction - a critical review. MLTJ 2017;7(2):230-9.

25. Mathis DT, Rasch H, Hirschmann MT. In vivo bone tunnel remodeling in symptomatic patients after ACL reconstruction: a retrospective comparison of articular and extra-articular fixation. MLTJ 2016;5(4):316-24.

26. Price R, Stoney J, Brown G. Prospective randomized comparison of endobutton versus cross-pin femoral fixation in hamstring anterior cruciate ligament reconstruction with 2-year follow-up. ANZ J Surg 2010;80(3):162-5.

27. Asik M, Sen C, Tuncay I, Erdil M, Avci C, Taser OF. The midto long-term results of the anterior cruciate ligament reconstruction with hamstring tendons using Transfix technique. Knee Surg Sports Traumatol Arthrosc 2007;15(8):965-72.

28. Barrow AE, Pilia M, Guda T, Kadrmas WR, Burns TC. Femoral Suspension Devices for Anterior Cruciate Ligament Reconstruction: Do Adjustable Loops Lengthen? Am J Sports Med 2014;42(2):343-9.

29. Hasan S, Nayyar S, Onyekwelu I, Kalra K, Gyftopoulos S, Jazrawi LM. Complications Using Bioabsorbable Cross-Pin Femoral Fixation: A Case Report and Review of the Literature. Case Rep Radiol 2011;2011:1-6.

30. Padulo J, Oliva F, Frizziero A, Maffulli N. Muscles, Ligaments and Tendons Journal - Basic principles and recommendations in clinical and field Science Research: 2018 update. MLTJ 2018;8(3):305-307. 